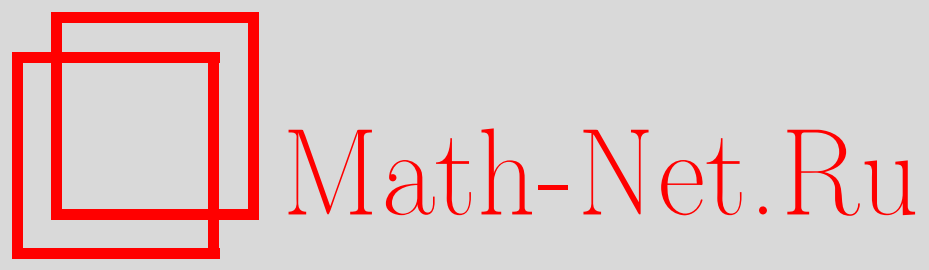

Ю. М. Березанский, Пуассонов бесконечномерный анализ как пример анализа, связанного с операторами обобщенного сдвига, Функи. анализ и его прил., 1998, том 32, выпуск 3, 65-70

DOI: https://doi.org/10.4213/faa424

Использование Общероссийского математического портала MathNet.Ru подразумевает, что вы прочитали и согласны с пользовательским соглашением

http://www . mathnet.ru/rus/agreement

Параметры загрузки:

IP : 54.237 .59 .107

26 апреля 2023 г., 09:34:26

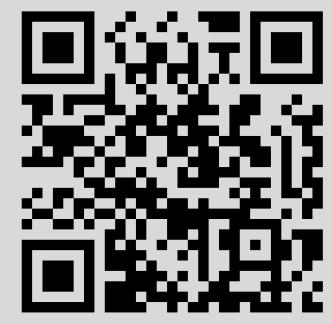


где $\left(a_{i}^{*}\right)$ - перестановка последовательности $\left(\left|a_{k}\right|\right)_{k=1}^{\infty}$ в убывающем порядке.

ЗАмЕчАНиЕ 2. Теоремы 2 и 3 усиливают результаты работы [2], где аналогичные утверждения были получены для пространств последовательностей $F$, удовлетворяющих более ограничительным условиям. Так, например, нетрудно показать, что норма оператора растяжения

$$
\sigma_{n} a=(\underbrace{a_{1}, \ldots, a_{1}}_{n}, \underbrace{a_{2}, \ldots, a_{2}}_{n}, \ldots)
$$

в пространстве $l_{1}(\ln )$ (см. пример 2) равна $n$. Поэтому условие (11) из [2] для этого пространства не выполнено, и, значит, к нему не применимы имеющиеся там теоремы.

ЗАмечАниЕ 3. Теоремы 1-3 легко переносятся на лакунарные тригонометрические ряды с помощью результатов [9].

\title{
ЛИТЕРАТУРА
}

1. Крейн С. Г., Петунин Ю. И., Семенов Е. М. Интерполяция линейных опеpaторов. Наука, M., 1978. 2. Rodin V. A., Semenov E. M. Analysis Math., 1, No. 3, 207-222 (1975). 3. Трибель $X$. Теория интерполяции, функциональные пространства, дифференциальные операторы. Мир, М., 1980. 4. Wallsten R. Lect. Notes Math., Vol. 1302, 410-419 (1988). 5 Родин B. А., Семенов E. M. Функц. анализ и его прил., 13, вып. 2, 91-92 (1979). 6. Montgomery-Smith S. Proc. Am. Math. Soc., 109, No. 2, 517-522 (1990). 7. Берг Й., Лефстрем Й. Интерполяционные пространства. Введение. Мир, М., 1980. 8. Calton N. J. Calderon couples of rearrangement invariant spaces. Preprint, 1993. 9. Jakubowski J., Kwapien S. Bull. Polish Acad. Sci. Math., 27, No. 9, 689-694 (1979).

Самарский госуниверситет

e-mail: astashkn@ssu.samara.emnet.ru

Поступило в редакцию 7 октября 1996 г.

УДК 517.515

\section{Пуассонов бесконечномерный анализ как пример анализа, связанного с операторами обобщенного сдвига}

\author{
(c) 1998. Ю. М. БЕРЕЗАНСКИЙ
}

Пуассонов бесконечномерный анализ, т.е. аналог классического анализа белого шума в том случае, когда мера, задающая спаривание, является не гауссовой, а пуассоновой, был построен и изучался в ряде работ [1-3]. В $[4,5]$ автор предложил общую схему построения бесконечномерного анализа, осно-

^ Работа частично поддержана ДФФД Украины, грант 1.4/62. 
ванную на понятии оператора обобщенного сдвига. В заметке показывается, что пуассонов анализ вкладывается в эту общую схему. Она приводит также к ряду новых фактов в пуассоновом анализе, связанных с понятием характеров Дельсарта. В доказательствах существенно используется спектральный подход $[6,7]$. Для простоты мы рассматриваем лишь пуассонову меру с интенсивностью, равной лебеговой мере на оси. Отметим, что последних исследований по пуассонову анализу [8], связанных с геометрией пуассонова пространства, мы в этой заметке не касаемся.

1. Рассмотрим классическую вещественную цепочку $\mathscr{S}_{\operatorname{Re}}^{\prime} \supset L_{\operatorname{Re}}^{2} \supset \mathscr{S}_{\operatorname{Re}}$, где $L_{\mathrm{Re}}^{2}=: N_{0, \operatorname{Re}}$ построено по лебеговой мере $d t$ на оси $\mathbb{R}^{1}$, а $\mathscr{S}_{\mathrm{Re}}-$ пространство Шварца; соответствующее спаривание будем обозначать через $\langle\cdot, \cdot\rangle$. Мера Пуассона $\pi$ на $\mathscr{S}_{\operatorname{Re}}^{\prime}$ с интенсивностью $d t$ определяется в силу теоремы Минлоса своим преобразованием Фурье

$$
\int_{\mathscr{S}_{\mathrm{Re}}^{\prime}} e^{i\langle\lambda, \xi\rangle} d \pi(\xi)=\exp \left\langle 1, e^{i \lambda}-1\right\rangle, \quad \lambda \in \mathscr{S}_{\mathrm{Re}}, \pi\left(\mathscr{S}_{\mathrm{Re}}^{\prime}\right)=1
$$

Меру $\pi$ можно сузить на гильбертово пространство и понимать ее как вероятностную меру на негативном пространстве $N_{-1, \operatorname{Re}} \subset \mathscr{S}_{\mathrm{Re}}^{\prime}$ вещественной цепочки $N_{-1, \operatorname{Re}} \supset N_{0, \operatorname{Re}} \supset N_{1, \operatorname{Re}}$, где $N_{1, \operatorname{Re}}=W_{2, \operatorname{Re}}^{2}\left(\mathbb{R}^{1},\left(1+t^{2}\right)^{2} d t\right)-$ соболевское пространство с весом $\left(N_{-1, R e}\right.$ - пространство полной меры $\pi$,

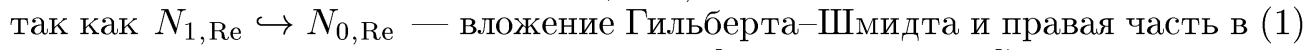
непрерывна относительно $\lambda \in N_{1, \operatorname{Re}}$; см. [9, Ch. $1, \S 3$, n. 8]).

Более того, нам удобно сузить $\pi$ до вероятностной меры на множестве


такой конфигурацией $x$ будем понимать множество пар $x=\left\{\left(t_{1}, \nu_{1}\right),\left(t_{2}, \nu_{2}\right)\right.$, $\ldots\}$, где $t_{1}, t_{2}, \ldots$ - не более чем счетное множество различных точек из $\mathbb{R}^{1}$, конечное на каждом конечном интервале, а $\nu_{k} \in \mathbb{Z}_{+}=\{0,1, \ldots\}$ - «кратность» вхождения точки $t_{k}$ в $x,\left(t_{k}, 0\right)=\varnothing$. Каждую кратную конфигурацию $x$ можно отождествить с обобщенной функцией $x \in \mathscr{D}_{\operatorname{Re}}^{\prime} \supset$ $\mathscr{S}_{\operatorname{Re}}^{\prime} \supset N_{-1, \operatorname{Re}}\left(\mathscr{D}_{\operatorname{Re}}=C_{0, \operatorname{Re}}^{\infty}\left(\mathbb{R}^{1}\right)\right)$, если положить $x=\sum_{k=1}^{\infty} \nu_{k} \delta_{t_{k}}$, где $\delta_{t}$ есть $\delta$-функция, сосредоточенная в точке $t$. Ясно, что $\delta_{t}$ является примером кратной конфигурации.

Обозначим через $Q$ совокупность всех кратных конфигураций $x$, входя-

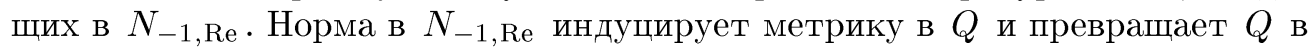
метрическое сепарабельное полное пространство. На $\sigma$-алгебре борелевских множеств из $Q$ определена мера $\pi$ как сужение этой меры с $N_{-1, \operatorname{Re}}$. Пространство $Q$ будет пространством полной меры, т.е. $\pi(Q)=1$ (это следует из того, что меньшее множество обычных конфигураций, т. е. точек $x$, для которых $\nu_{1}=\nu_{2}=\cdots=1$, является множеством полной меры; см., напримep, [8]).

Таким образом, определено пространство $Q$ и пуассонова мера $\pi$ на нем. Ниже будут рассматриваться пространства основных и обобшенных функций переменной $x \in Q$ с комплексным спариванием относительно $\pi$, т.е. относительно $\left(L^{2}\right):=L^{2}(Q, d \pi(x))$. Всюду используется терминология и результаты заметки [4] (и ее подробного изложения [5]). 
2. Для введения операторов обобщенного сдвига заметим, что $Q$ является коммутативной полугруппой с операцией сложения + , индуцированной сложением в $N_{-1, \operatorname{Re}} \supset Q$; ее базисной единицей $e$ служит $\varnothing$ (иными словами, $Q \times Q \ni\{x, y\} \mapsto x+y:=x \cup y \in Q$, при этом кратности складываются для каждой общей точки $t_{k}$, фигурирующей в $x$ и $y$ ).

В линейном пространстве $C(Q)$ всех комплекснозначных локально ограниченных непрерывных функций $f(x)$ на $Q$ введем оператор обобщенного сдвига $T_{x}$ для $x \in Q$, полагая $\left(T_{x} f\right)(y)=f(y+x), y \in Q$. Очевидно, что все требования на семейство $T=\left\{T_{x}\right\}_{x \in Q}$ будут выполняться.

3. Роль пространств $N_{p}$ комплексной цепочки $N_{-p} \supset N_{0} \supset N_{p}, p \in \mathbb{N}$, в построениях $[4,5]$ будут играть соболевские пространства $N_{p}:=$ $W_{2}^{1+p}\left(\mathbb{R}^{1},\left(1+t^{2}\right)^{1+p} d t\right)$. Вложение $N_{2} \hookrightarrow N_{1}$ будет вложением ГильбертаШмидта согласно [9]; пространство $N_{p}^{\widehat{\otimes} n}$ состоит из симметрических функций $f^{n}\left(t_{1}, \ldots, t_{n}\right), t_{k} \in \mathbb{R}^{1}$, с достаточной гладкостью и убыванием на $\infty$.

В качестве характеров примем функции

$$
Q \ni x \mapsto \chi(x, \lambda)=\exp \langle x, \log (1+\lambda)\rangle \in \mathbb{C}^{1},
$$

зависящие от параметра $\lambda \in B_{1}=\left\{\lambda \in N_{1}:\|\lambda\|_{N_{1}} \leqslant R_{1}\right\}$, где $R_{1}>0$ достаточно мало. Поясним, что справедлива оценка $\left(1+t^{2}\right)^{1 / 2}|\lambda(t)| \leqslant c_{1}\|\lambda\|_{N_{1}}$, $t \in \mathbb{R}^{1}$, с некоторым $c_{1}>0$ [9]. Поэтому $1+\lambda(t) \neq 0$ при достаточно малой $\|\lambda\|_{N_{1}}$ и формула $(2)$ имеет смысл, а функция $\chi(x, \lambda)$ является аналитической относительно $\lambda$ и локально ограниченной относительно $x$. Таким образом, для каждого $x \in Q$ справедливо разложение в ряд при достаточно малом $R_{2}>0$ :

$$
\chi(x, \lambda)=\sum_{n=0}^{\infty} \frac{1}{n !}\left\langle\lambda^{\otimes n}, \chi_{n}(x)\right\rangle, \quad \lambda \in B_{2}=\left\{\lambda \in N_{2}:\|\lambda\|_{N_{2}} \leqslant R_{2}\right\},
$$

где $Q \ni x \mapsto \chi_{n}(x) \in N_{-2, \mathrm{Re}}^{\widehat{\otimes} n} \subset N_{-2}^{\widehat{\otimes} n}-$ характеры Дельсарта. Эти характеры несложно подсчитываются; они являются сужением на $Q$ некоторых полиномов переменной $x \in N_{-2, \operatorname{Re}} \supset Q$.

Теорема 1. Справедлива следующая оченка: существует $C>0$, такое, чmo

$$
\|\| \chi_{n}(\cdot)\left\|_{N_{-2}^{\widehat{\otimes} n}}\right\|_{\left(L^{2}\right)} \leqslant C^{n} n !, \quad n \in \mathbb{Z}_{+} .
$$

Элементарнье функции $Q \ni x \mapsto\left\langle a^{n}, \chi_{n}(x)\right\rangle \in \mathbb{C}^{1} n p u a^{n} \in N_{2}^{\widehat{\otimes} n}($ входяшие в силу (4) в $\left(L^{2}\right)$ ) линейно независимь, образуют в требуемом смьсле минимальную систему, и их линейная оболочка плотна в $\left(L^{2}\right)$.

4. Для введения «классических» характеров Аппеля рассмотрим функцию

$$
\begin{gathered}
Q \ni x \mapsto \sigma(x, \lambda)=\exp (\langle x, \log (1+\lambda)\rangle-\langle 1, \lambda\rangle)=\chi(x, \lambda)(\widehat{\pi}(\lambda))^{-1} \in \mathbb{C}^{1}, \\
\widehat{\pi}(\lambda)=\int_{Q} \chi(x, \lambda) d \pi(x)=\exp \langle 1, \lambda\rangle .
\end{gathered}
$$


Она аналитична относительно $\lambda \in N_{1}$ в той же области, что и $\chi(x, \lambda)$, и поэтому допускает разложение

$$
\sigma(x, \lambda)=\sum_{n=0}^{\infty} \frac{1}{n !}\left\langle\lambda^{\otimes n}, P_{n}(x)\right\rangle, \quad \lambda \in B_{2},
$$

подобное (3). Его коэффициенты $Q \ni x \mapsto P_{n}(x) \in N_{-2, \operatorname{Re}}^{\widehat{\otimes} n} \subset N_{-2}^{\widehat{\otimes} n}$ (т.е. характеры Аппеля) в данном случае носят название (обобщенных) полиномов Шарлье. Для них сохраняется оценка типа (4). Более того, она усиливается: в действительности $P_{n}(x) \in N_{-1, \operatorname{Re}}^{\widehat{\otimes} n} \subset N_{-1}^{\widehat{\otimes} n}, x \in Q$, для некоторого $D>0$

$$
\|\| P_{n}(\cdot)\left\|_{N_{-1}^{\widehat{\otimes} n}}\right\|_{\left(L^{2}\right)} \leqslant D^{n} \sqrt{n !}, \quad n \in \mathbb{Z}_{+} .
$$

5. Таким образом, сейчас возможно построение пространств основных и обобщенных функций по характерам Аппеля (т.е. по полиномам Шарлье) и Дельсарта и соответствующего пуассонова анализа. Так, позитивное пространство $H^{P}(p, q)$, построенное по полиномам Аппеля, имеет вид

$$
\begin{aligned}
H^{P}(p, q)=\left\{\varphi(x)=\sum_{n=0}^{\infty}\left\langle a^{n}, P_{n}(x)\right\rangle, a^{n} \in N_{p}^{\widehat{\otimes} n} \mid\right. \\
\left.\|\varphi\|_{H^{P}(p, q)}^{2}=\sum_{n=0}^{\infty}\left\|a^{n}\right\|_{N_{p}^{\widehat{\otimes} n}}^{2}(n !)^{2} K^{q n}<\infty\right\},
\end{aligned}
$$

где фиксированы $p \in \mathbb{N}_{1}:=\{2,3, \ldots\}, q \in \mathbb{N}$ и достаточно большое $K>1$. Аналогично строится пространство $H^{\chi}(p, q)$ : нужно в (7) заменить $P_{n}$ на $\chi_{n}$.

Оператор уничтожения $\partial\left(\alpha^{m}\right), \alpha^{m} \in N_{-p}^{\widehat{\otimes} m}$, әде $m \in \mathbb{Z}_{+} u p \in \mathbb{N}_{1}[4,5]$, в пуассоновом анализе легко подсчитывается. В самом деле, для финитного $a^{m} \in N_{p}^{\widehat{\otimes} m}$ введем на функциях $f \in C(Q)$ разностную операцию

$$
\begin{gathered}
\left(\mathscr{L}\left(a^{m}\right) f(\cdot)\right)(x)=\int_{\mathbb{R}^{m}}\left(\mathscr{L}\left(\delta_{t_{1}}\right) \cdots \mathscr{L}\left(\delta_{t_{m}}\right) f(\cdot)\right)(x) a^{m}\left(t_{1}, \ldots, t_{m}\right) d t_{1} \cdots d t_{m}, \\
\left(\mathscr{L}\left(\delta_{t}\right) f(\cdot)\right)(x)=f\left(x+\delta_{t}\right)-f(x) \quad\left(x \in Q, t \in \mathbb{R}^{1}\right) .
\end{gathered}
$$

Применяя (8) к характеру (2), убеждаемся, что

$$
\left(\mathscr{L}\left(a^{m}\right) \chi(\cdot, \lambda)\right)(x)=\left\langle a^{m}, \lambda^{\otimes m}\right\rangle \chi(x, \lambda)
$$

для $x \in Q$ и $\lambda \in B_{1}$. Таким образом, сейчас $T_{x}$ является сдвигом ТейлораДельсарта, и поэтому для финитных $a^{m} \in N_{p}^{\widehat{\otimes} m}$

$$
\partial\left(a^{m}\right)=\mathscr{L}\left(a^{m}\right) \quad\left(p \in \mathbb{N}_{1}, m \in \mathbb{Z}_{+}\right) .
$$

Наконец, оператор, заданный формулами (9), (8), по непрерывности распространяется до оператора $\partial\left(\alpha^{m}\right)$ nри $\alpha^{m} \in N_{-p}^{\widehat{\otimes} m}$, действуючего в пространствах $H^{\chi}(p, q)$ и $H^{P}(p, q)\left(p \in \mathbb{N}_{1}, q \in \mathbb{N}\right)$. 
Теорема 2. Результаты по бесконечномерному анализу, содержащиеся в [4, 5], справедливь для пуассонова анализа с введенным выие пространством $Q$, пуассоновой мерой $\rho=\pi$, сдвигом $\left(T_{x} f\right)(y)=f(y+x)(x, y \in Q)$ и характерами Дельсарта и Аппеля, задаюшимися разложениями (3), (5). Onepamop $\partial\left(\alpha^{m}\right)\left(\alpha^{m} \in N_{-p}^{\widehat{\otimes} m}, p \in \mathbb{N}_{1}, m \in \mathbb{Z}_{+}\right)$имеет вид $(9),(8)$.

При этом характерьл $P_{n}(x)$ и кохарактерь $Q\left(\alpha^{n}\right)$ Anпеля сейчас совпадают в том смисле, что $Q\left(a^{n}\right) \in C(Q)$ при $a^{n} \in N_{p}^{\widehat{\otimes} n}, p \in \mathbb{N}_{1}, n \in \mathbb{Z}_{+}, u$ справедливо равенство $\left(Q\left(a^{n}\right)\right)(x)=\left\langle a^{n}, P_{n}(x)\right\rangle, x \in Q$.

Таким образом, полиномь Шарлье удовлетворяют соотношению ортогональности

$$
\begin{gathered}
\int_{Q}\left\langle a^{n}, P_{n}(x)\right\rangle \overline{\left\langle b^{m}, P_{m}(x)\right\rangle} d \pi(x)=\delta_{n, m} n !\left\langle a^{n}, \overline{b^{m}}\right\rangle \\
\left(a^{n} \in N_{p}^{\widehat{\otimes} n}, b^{m} \in N_{r}^{\widehat{\otimes} m}, p, r \in \mathbb{N}_{1}, n, m \in \mathbb{Z}_{+}\right),
\end{gathered}
$$

и для них справедлива формула $\left\langle a^{n}, P_{n}(\cdot)\right\rangle=\partial^{+}\left(\overline{a^{n}}\right) 1$, где + означает сопряжение относительно пространства $\left(L^{2}\right)$.

6. Для доказательства теорем 1, 2 сушественную роль играет спектральный подход к пуассонову анализу [6, 7], заключающийся в следующем.

В симметричном пространстве Фока $\mathscr{F}(H)=\bigoplus_{n=0}^{\infty} \mathscr{F}_{n}(H), H=L_{\mathrm{Re}}^{2}=$ $N_{0, R e}$, рассматривается якобиево поле самосопряженных коммутирующих операторов $A=(A(\varphi))_{\varphi \in \mathscr{S}_{\mathrm{Re}}}, A(\varphi)=A_{0}(\varphi)+B(\varphi)+\langle\varphi, 1\rangle \mathrm{Id}$, где $A_{0}(\varphi)$ - классический полевой оператор, а $B(\varphi)$ - его возмущение диагональным оператором, равным вторичному квантованию оператора умножения на $\varphi$ в пространстве $L^{2}$.

Преобразование Фурье по обобщенным совместным собственным векторам семейства $A$ имеет вид [7]

$$
\mathscr{F}(H) \supset \mathscr{F}\left(H_{1}, p\right) \ni f=\left(f^{n}\right)_{n=0}^{\infty} \mapsto \hat{f}(x)=\sum_{n=0}^{\infty}\left\langle f^{n}, \mathscr{P}_{n}(x)\right\rangle \in\left(L^{2}\right),
$$

где $\mathscr{F}\left(H_{1}, p\right)$ - взвешенное посредством некоторого веса $p$ пространство Фока, построенное по $H_{1}=N_{1}$, Rе $\subset H$, а $\left(\mathscr{P}_{n}(x)\right)_{n=0}^{\infty}-$ связанная с $A$ последовательность «ортонормированных» полиномов первого рода $Q \ni x \mapsto$ $\mathscr{P}_{n}(x) \in N_{-1}^{\widehat{\otimes} n} \subset N_{-2}^{\widehat{\otimes} n}$. Спектральной мерой поля $A$ служит мера $\pi$ на $Q$. Отображение (10) после замыкания по непрерывности превращается в изометрию между $\mathscr{F}(H)$ и $\left(L^{2}\right)$.

Справедливо равенство $P_{n}(x)=\sqrt{n !} \mathscr{P}_{n}(x), n \in \mathbb{Z}_{+}, x \in Q$ (с его помощью устанавливается (6) и теорема 1$)$. Полагая $a^{n}=(n !)^{-1 / 2} f^{n}$, получим, что (10) определяет изометрию $\mathscr{U}$ между пространством Фока $F(H)$ с нормой $\|a\|_{F(H)}^{2}=\sum_{n=0}^{\infty}\left\|a^{n}\right\|_{N_{0}^{\widehat{\otimes} n}}^{2} n !$ и пространством $\left(L^{2}\right)$ функций $(\mathscr{U} a)(x)=$ $\sum_{n=0}^{\infty}\left\langle a^{n}, P_{n}(x)\right\rangle$. Беря различные оснащения такого пространства Фока и рассматривая их $\mathscr{U}$-образы, получим оснашения пространства $\left(L^{2}\right)((7)-$ пример такого оснащения). 
Отметим, что операторы $\partial\left(a^{1}\right)$ и $\partial^{+}\left(a^{1}\right)$ являются $\mathscr{U}$-образами классических операторов уничтожения и рождения, порожденных вектором $a^{1} \in H$, но записанных в терминах $F(H)$, а не $\mathscr{F}(H)$.

Автор благодарен профессору Г. Ф. Усу и доктору Н. А. Качановскому за обсуждение и полезные замечания.

\section{ЛитеРАтУРА}

1. Ito $Y$. Probab. Theory Related Fields, 77, 1-28 (1988). 2. Ito Y., Kubo I. Nagoya Math. J., 111, 41-84 (1988). 3. Kondratiev Yu. G., da Silva J. L., Streit L., Us G. F. Infinite Dim. Anal. Quantum Prob. Related Topics, 1, No. 1, 91-117 (1998). 4. Бepeзанский Ю. М. Функц. анализ и его прил., 30, вып. 4, 61-65 (1996). 5. Березанский Ю. М. Укр. мат. ж., 49, № 3, 364-409 (1997). 6. Lytvynov E. W. Methods Funct. Anal. Topology, 1, No. 1, 61-85 (1995). 7. Berezansky Yu. M. Integral Equations Operator Theory, 30, No. 2, 163-190 (1998). 8. Albeverio S., Kondratiev Yu. G., Röckner $M$. Analysis and geometry on configuration spaces, Bielefeld, Preprint BiBoS, 1997. 9. Berezansky Yu. M. Selfadjoint operators in space of functions of infinitely many variables. AMS, Providence, RI, 1986.

Институт математики НАНУ,

Киев, Украина

Поступило в редакцию

e-mail: berezan@mathber.carrier.kiev.ua 14 мая 1998 г.

УдК 517.956

\section{Спектральные асимптотики операторов Лапласа на каспе: случай быстро растущей считающей функции}

(C) 1998. С. И. БОяРчЕнко, С. З. ЛЕВЕНДОРСКИй

1. Пусть $\Omega^{\prime} \subset \mathbb{R}^{n-1}$ - ограниченная область, и пусть $f: \overline{\mathbb{R}}_{+} \rightarrow \mathbb{R}_{+}$растет на бесконечности:

$$
f(t) \rightarrow+\infty \text { при } t \rightarrow+\infty .
$$

Рассмотрим операторы Дирихле $-\Delta_{D}^{\Omega}$ и Лапласа $-\Delta_{N}^{\Omega}$ в каспидальной области $\Omega=\left\{(t, x) \mid t>0, f(t) x \in \Omega^{\prime}\right\}$. Оператор Дирихле есть оператор в $L_{2}(\Omega)$, такой, что

$$
\left\langle-\Delta_{D}^{\Omega} u, u\right\rangle=\int_{\Omega}\left|\nabla_{x} u\right|^{2} d x
$$

и $C_{0}^{\infty}(\Omega)$ - ядро квадратичной формы; оператор Неймана определяется аналогично, но с $C_{0}^{\infty}(\bar{\Omega})$ вместо $C_{0}^{\infty}(\Omega)$.

Нетрудно видеть, что если выполняется условие (1), то оператор Лапласа $-\Delta_{D}^{\Omega}$ является самосопряженным положительно определенным оператором с

\footnotetext{
^ Исследование поддержано РФФИ, грант 96-01-01196.
} 